\title{
KRULL VERSUS GLOBAL DIMENSION IN NOETHERIAN P.I. RINGS
}

\author{
K. R. GOODEARL AND L. W. SMALL ${ }^{1}$
}

\begin{abstract}
The Krull dimension of any noetherian P.I. ring is bounded above by its global (homological) dimension (when finite).
\end{abstract}

1. Introduction. A longstanding open problem is the conjecture that for a noetherian ring $R$ of finite global dimension, the Krull dimension of $R$ is no larger than the global dimension. This conjecture was verified for semiprime noetherian P.I. rings by Resco, Small, and Stafford [7, Theorem 3.2], and more recently for certain fully bounded noetherian rings by Brown and Warfield [2, Corollary 12], as follows.

THEOREM A. (BROWN-WARFIELD). Let $R$ be a fully bounded noetherian ring containing an uncountable set $F$ of central units such that the difference of any two distinct elements of $F$ is a unit. If gl.dim. $(R)$ is finite, then $\mathrm{K} . \operatorname{dim} .(R) \leqslant \operatorname{gl} \cdot \operatorname{dim} .(R)$.

We proceed by applying Theorem A to Laurent series rings, using the following observation.

Proposition B. If $R$ is a nonzero noetherian P.I. ring, then the Laurent series ring $R((x))$ is a fully bounded noetherian ring containing an uncountable set $F$ of central units such that the difference of any two distinct elements of $F$ is a unit.

Proof. Set $T=R((x)$ ); it is well known that $T$ is noetherian (or see Proposition 2).

By [6, Theorem II.4.1], $R$ satisfies a multilinear identity $f$ with coefficients \pm 1 . Then $f$ is satisfied in $R[x]$, and hence in $R[x] / x^{n} R[x]$, for all positive integers $n$. As $R[[x]]$ is an inverse limit of the rings $R[x] / x^{n} R[x]$, it satisfies $f$, whence $T$, being a central localization of $R[[x]]$, satisfies $f$. Thus $T$ is a P.I. ring. By [1, Theorem 7 or 6 , Theorem II.5.3], $T$ is fully bounded.

Let $F$ be the set of those Laurent series in $T$ with all coefficients either 0 or 1 , and note that $F$ is an uncountable subset of the center of $T$. Any element of $F$, or any difference of two distinct elements of $F$, has leading coefficient \pm 1 and so is a unit in $T$.

Received by the editors October 24, 1983.

1980 Mathematics Subject Classification. Primary 16A33, 16A38, 16A55, 16A60.

Key words and phrases. Noetherian ring, polynomial identity, Krull dimension, homological dimension.

${ }^{1}$ The research of both authors was partially supported by grants from the National Science Foundation. 
To obtain our general result requires the following change of rings theorem, proved in $\S 2$.

THEOREM C. If $R$ is a right noetherian ring and $T$ is the Laurent series ring $R((x))$, then r.K.dim. $(R)=$ r.K.dim. $(T)$ and r.gl.dim. $(R)=$ r.gl.dim. $(T)$.

Our main result is now an immediate consequence of Theorems $\mathrm{A}$ and $\mathrm{C}$ and Proposition B.

THEOREM D. If $R$ is any noetherian P.I. ring for which $\operatorname{gl} . \operatorname{dim} .(R)$ is finite, then K.dim. $(R) \leqslant \operatorname{gl} . \operatorname{dim} .(R)$.

2. Laurent series rings. Here we consider the Krull and global dimensions of Laurent series rings over arbitrary noetherian rings (not necessarily P.I.).

Definition. Let $T=R((x))$ be the Laurent series ring over a ring $R$. Any nonzero element $t \in T$ has the form

$$
t=\sum_{i=n}^{\infty} t_{i} x^{i},
$$

where $n \in \mathbf{Z}$, each $t_{i} \in R$ and $t_{n} \neq 0$. The integer $n$ is called the order of $t$, and the element $t_{n}$ is called the leading coefficient of $t$, which we shall denote by $\lambda(t)$. By convention, $\lambda(0)=0$. For a right ideal $I$ of $T$, define $\lambda(I)=\{\lambda(t) \mid t \in I\}$, and observe that $\lambda(I)$ is a right ideal of $R$.

LEMMA 1. Let $R$ be a ring, let $T=R((x))$ and let $I, J$ be right ideals of $T$ such that $I \subseteq J$. If $\lambda(I)=\lambda(J)$ and $\lambda(I)$ is finitely generated, then $I=J$.

Proof. We may assume that $I \neq 0$. Choose nonzero elements $a_{1}, \ldots, a_{m}$ in $I$ such that $\lambda\left(a_{1}\right), \ldots, \lambda\left(a_{m}\right)$ generate $\lambda(I)$. After multiplying the $a_{i}$ by suitable powers of $x$, we may assume that the $a_{i}$ all have order 0 .

Now consider any nonzero element $b \in J$. In showing that $b \in I$, there is no harm in multiplying $b$ by a power of $x$. Hence, we may assume that $b$ has order 0 . We construct elements $s_{i j} \in R$ (for $i=1, \ldots, m$ and $j=0,1,2, \ldots$ ) such that for each $n=0,1,2, \ldots$, the element

$$
b-\sum_{i=1}^{m} \sum_{j=0}^{n} a_{i} s_{i j} x^{j}
$$

has order greater than $n$.

Since $\lambda(b) \in \lambda(J)=\lambda(I)=\Sigma \lambda\left(a_{i}\right) R$, there exist elements $s_{i 0} \in R$ such that

$$
\lambda(b)=\lambda\left(a_{1}\right) s_{10}+\cdots+\lambda\left(a_{m}\right) s_{m 0} .
$$

As $a_{1}, \ldots, a_{m}, b$ all have order 0 , the element

$$
b-\left(a_{1} s_{10}+\cdots+a_{m} s_{m 0}\right)
$$

must have order greater than 0 .

Now assume that we have constructed $s_{i j} \in R$ for $i=1, \ldots, m$ and $j=0,1, \ldots, n$ such that the element

$$
c=b-\sum_{i=1}^{m} \sum_{j=0}^{n} a_{i} s_{i j} x^{j}
$$


has order greater than $n$. Note that $c \in J$, and let $c_{n+1}$ denote the coefficient of $x^{n+1}$ in $c$. Either $c_{n+1}=0$ or $c_{n+1}=\lambda(c)$, whence $c_{n+1} \in \lambda(J)$ in either case. There exist elements $s_{i, n+1} \in R$ such that

$$
c_{n+1}=\lambda\left(a_{1}\right) s_{1, n+1}+\cdots+\lambda\left(a_{m}\right) s_{m, n+1},
$$

and the element

$$
c-\left(a_{1} s_{1, n+1} x^{n+1}+\cdots+a_{m} s_{m, n+1} x^{n+1}\right)
$$

has order greater than $n+1$. This completes the induction step.

Finally, setting $d_{i}=\sum_{j=0}^{\infty} s_{i j} x^{j}$ for each $i=1, \ldots, m$, we conclude that $b=a_{1} d_{1}$ $+\cdots+a_{m} d_{m}$. Therefore $b \in I$.

Proposition 2. Let $R$ be a right noetherian ring, and let $T=R((x))$. Then $T$ is a right noetherian ring and r.K.dim. $(T)=$ r.K.dim. $(R)$.

Proof. We have a map $\lambda$ from the lattice of right ideals of $T$ to the lattice of right ideals of $R$, and Lemma 1 shows that $\lambda$ preserves strict inclusions. Consequently, $T$ is right noetherian, and r.K.dim. $(T) \leqslant \mathrm{r}$.K.dim. $(R)$.

For each right ideal $I$ of $R$, let $I((x))$ denote the right ideal of $T$ consisting of those elements of $T$ with all coefficients lying in $I$. The map $I \mapsto I((x))$ defines an embedding of the lattice of right ideals of $R$ into the lattice of right ideals of $T$, whence r.K.dim. $(R) \leqslant \mathrm{r} . \mathrm{K} . \operatorname{dim} .(T)$.

THEOREM 3. Let $R$ be a right noetherian ring, and let $T=R((x))$. Then r.gl.dim. $(T)$ $=$ r.gl.dim. $(R)$.

Proof. Since $R$ is right coherent, all direct products of flat left $R$-modules are flat [3, Theorem 2.1]. Hence, for each integer $n$, the set $T_{n}$ consisting of those elements of $T$ with order at least $n$ is a flat left $R$-module. Thus $T$, being the union of the $T_{n}$, is flat as a left $R$-module. In addition, $R$ is an $(R, R)$-bimodule direct summand of $T$. Therefore r.gl.dim. $(R) \leqslant \mathrm{r}$.gl.dim $(T)$, by $[5$, Lemma 1$]$.

We may now assume that r.gl.dim. $(R)=n<\infty$. Set $S=R[[x]]$. Since $x$ is a central regular element in the Jacobson radical of $S$ and $S / x S \cong R$, [4, Part III, Theorem 10] shows that r.gl.dim $(S)=n+1$. On the other hand, r.gl.dim. $(T) \leqslant$ r.gl.dim $(S)$ because $T$ is a central localization of $S$. Thus r.gl.dim.( $T)$ equals either $n$ or $n+1$.

If $\operatorname{rgl} \operatorname{dim}(T)=n+1$, there exists a right ideal $I$ in $T$ such that $T / I$ has projective dimension $n+1$. Set $J=I \cap S$ and $A=S / J$, and observe that $A \otimes_{S} T$ $\cong T / I$. Now $A$ is a finitely generated right $S$-module on which $x$ is a non-zerodivisor, and we observe that $A$ must have projective dimension $n+1$. According to [4, Part III, Theorem 9], the right $R$-module $A / A x$ must have projective dimension $n+1$, which is impossible.

Therefore r.gl.dim. $(T)=n$.

\section{REFERENCES}

1. S. A. Amitsur, Prime rings having polynomial identities with arbitrary coefficients, Proc. London Math. Soc. (3) 17 (1967), 470-486.

2. K. A. Brown and R. B. Warfield, Jr., Krull and global dimensions of fully bounded noetherian rings, Proc. Amer. Math. Soc. 92 (1984), 169-174. 
3. S. U. Chase, Direct products of modules, Trans. Amer. Math. Soc. 97 (1960), 457-473.

4. I. Kaplansky, Fields and rings, Univ. of Chicago Press, Chicago, Ill., 1969.

5. J. C. McConnell, On the global dimension of some rings, Math. Z. 153 (1977), 253-254.

6. C. Procesi, Rings with polynomial identities, Dekker, New York, 1973.

7. R. Resco, L. W. Small and J. T. Stafford, Krull and global dimensions of semiprime noetherian PI-rings, Trans. Amer. Math. Soc. 274 (1982), 285-295.

Department of Mathematics, University of Utah, Salt Lake City, Utah 84112

Department of Mathematics, University of California - San Diego, la Jolla, California 92093 